\title{
Cementless Hemiarthroplasty Without Femoral Calcar Reconstruction Versus Cemented Hemiarthroplasty for Unstable Intertrochanteric Fractures in octogenarians
}

\author{
Chang Sun \\ Jinling Hospital, Nanjing University School of Medicine \\ Meng Cai \\ Jinling Hospital, School of Medicine, Southeast University \\ Jia Meng \\ Jinling Hospital, Nanjing University School of Medicine \\ Yu Zhang \\ Jinling Hospital, Nanjing University School of Medicine \\ Hui Jiang \\ Jinling Hospital, Nanjing University School of Medicine \\ Jianning Zhao \\ Jinling Hospital, Nanjing Universit School of Medicine \\ Ting Guo ( $\square$ guotingdoc@126.com ) \\ Jinling Hospital, Nanjing University School of Medicine
}

Research article

Keywords: unstable intertrochanteric fracture, cementless hemiarthroplasty, modular long stem, femoral calcar reconstruction

Posted Date: August 10th, 2021

DOI: https://doi.org/10.21203/rs.3.rs-775419/v1

License: (c) (i) This work is licensed under a Creative Commons Attribution 4.0 International License. Read Full License 


\section{Abstract \\ Background}

The aim of this study was to compare the mid-term outcomes of cementless hemiarthroplasty without femoral calcar reconstruction with cemented hemiarthroplasty in patients aged 80 years or more.

\section{Methods}

From January 2015 to December 2017, 135 patients with unstable intertrochanteric fractures were retrospectively investigated, 70 patients were treated with the cemented hemiarthroplasty while 65 patients were treated with a modular long-stem cementless hemiarthroplasty without rebuilding calcar. Clinical and radiological results were analyzed.

\section{Results}

The 106 alive patients were followed for $62.1 \pm 8.3$ months, and 29 patients died during the follow-up period. No significant differences were found in terms of demographic and clinical characters of patients, weight training time, postoperative hospital stay length, postoperative ambulatory status and Harris hip score. Patients treated with cementless hemiarthroplasty had a shorter operative time, less estimated blood loss, less amount of blood transfusion and a lower one-year mortality compared with patients treated with cemented hemiarthroplasty.

\section{Conclusions}

Compared with cemented hemiarthroplasty, cementless hemiarthroplasty using a long modular stem without reconstruction of femoral calcar was a good choice for octogenarians with unstable intertrochanteric fractures.

\section{Background}

With the aging trend of the population in the world, the incidence of hip fracture in elderly patients is steeply increasing[1]. Intertrochanteric fracture accounts for $50 \%$ of hip fracture, and the one-year mortality is $15 \%-20 \%[2]$. For the octogenarians with intertrochanteric fracture, they often have medical comorbidities such as hypertension, diabetes, chronic lung disease and poor bone quality with severe osteoporosis, so surgical treatment remains challenging. Currently surgical options have internal fixation and bipolar hemiarthroplasty. However, elderly patients with unstable intertrochanteric fracture and osteoporosis treated with internal fixation have high failure rates, ranging from $13-32 \%[3,4]$. And due to fear of loss of fixation and implant failure, patients are recommended to restrict early weight wearing and 
prolong bed rest, thus, subsequent morbidity and mortality are increased[5, 6]. Besides, the failure of internal fixation can cause more financial and risky cost for such patients[7].

Recently, hemiarthroplasty has been testified as a good choice for elderly patients with unstable intertrochanteric fracture to facilitate immediate postoperative weight wearing with low failure rate[7]. Studies have reported about clinical outcomes of cemented stems and cementless stems for unstable intertrochanteric fracture in elderly patients[8, 9]. Cemented stems have been used more frequently for reliable initial stability in elderly patients with osteoporosis, however, related studies showed that acrylic cement was associated with the increased risk of cardiopulmonary complications in elderly patients, and more time cost is needed for the preparation and seting of acrylic cement during the operation[10-12]. To replace the use of cemented stems, cementless stems need sufficient initial stabilities, so studies on different types of cementless stems such as calcar replacement, diaphyseal locking, and hydroxyapatitecoated long stem for unstable intertrochanteric fracture have been reported[9, 10,13].

Most studies are observational studies on cementless stems without control, Deniz Cankaya etc. retrospectively analyzed cemented and cementless hemiarthroplasty for intertrochanteric fractures accompanied with femoral calcar reconstruction in elderly patients with an average age of 77 years, which revealed cementless hemiarthroplasty is a reliable treatment with early mobilization, acceptable functional results, low implant loosening rates, shorter surgery time, lesser blood loss and lower perioperative mortality rate[14]. Femoral calcar reconstruction can strengthen initial instability and avoid subsidence of the femoral stem[15, 16], some studies suggest using calcar replacement instead of calcar reconstruction[13, 17]. However, calcar reconstruction or calcar replacement will increase the complexity of procedure, operative trauma and resultant complications, Zha etc. reported cementless hemiarthroplasty without calcar reconstruction achieved a satisfactory clinico-radiological outcome and regained preoperative ambulatory status in most patients (83.3\%)[18]. By now, there has been no casecontrol study comparing cementless stems without reestablishing femoral calcar and cemented stems for unstable intertrochanteric fracture in patients aged 80 years or more. Therefore, in this study we aimed to compare the mid-term survival reliability of cementless hemiarthroplasties without calcar reconstruction and cemented hemiarthroplasties for unstable intertrochanteric fractures in octogenarians.

\section{Methods}

We retrospectively reviewed 147 patients (147 hips) with unstable intertrochanteric fractures between January 2015 and December 2017, and institutional review board approval and related informed consent were obtained. The inclusion criteria were the follows: three parts or more fracture fragments with a loss of posteromedial cortical support, patients aged 80 years or more, surgical treatments with cemented or uncemented hemiarthroplasty. The exclusion criteria were the follows: pathological intertrochanteric fractures, previous contralateral hip fractures with decreased ambulatory abilities, multisite fractures. 12 patients were lost during the follow-up period, the rest 135 patients finished the investigation with 29 patients died. Among the eligible 135 patients, 70 patients underwent cemented hemiarthroplasty (Group 
A), the rest 65 patients underwent cementless hemiarthroplasty (Group B). All patients have at least two kinds of basic disease such as hypertension, diabetes, chronic lung disease, cardiovascular disease and so on, and American Society of Anesthesiologist (ASA) grades were recorded. Before the surgery, all patients underwent the comprehensive assessment which showed they can tolerate the hemiarthroplasty.

Demographic data including age at the time of surgery, gender, body mass index (BMI), ASA grade, EvansJensen classification of the intertrochanteric fracture, the Singh index for the osteoporosis[19] and the preoperative ambulatory status were obtained from medical records. Clinical evaluation was conducted by comparing the preoperative hemoglobin, the operative time, the estimated blood loss, blood transfusion, the length of hospital stays after surgery, postoperative complications, the Harris hip score (HHS), the postoperative mortality and the ambulatory status at the last follow-up. Radiographic evaluation including an assessment of the fixation, osteolysis and cortical porosis around femoral component, subsidence of the femoral component, heterotopic ossification and acetabular erosion was performed according to the radiological criteria described by Engh et al[20]. Cortical porosis was defined as a loss of endosteal definition with the somewhat sparse appearance of the remaining cortex[21]. Subsidence of the femoral component was measured according to the method described by Meijerink et al[22].

\section{Surgical Technique}

All patients were operated on via the posterolateral approach. In cemented group, the femoral head and neck were removed, the posteromedial fragments extending below the lesser trochanter were kept and reduced with bone cerclage. As for the posteromedial fragments not extending below the lesser trochanter, they were removed. The femoral canal was identified and reamed sequentially to achieve the final stem size. SP II femoral components (Waldemar Link, Hamburg, Germany) were used according to the preoperative X-ray measurement and the intraoperative measurement of the medullary cavity. In cementless group, the fractured fragments including head, neck, calcar and lesser trochanter were removed. The femoral canal was identified and reamed sequentially to achieve the final stem size. MP modular femoral components (Waldemar Link, Hamburg, Germany) were used according to the preoperative X-ray measurement and the intraoperative measurement of the medullary cavity. In both groups, the trial reduction was conducted with appropriate neck length and bipolar head diameter. For all patients, the anteversion of femoral component was set at 15-20区. If the greater trochanter fracture fragments were displaced, reductions were accomplished with cerclage wires. The posterior capsule and the short external rotators were routinely repaired.

On postoperative day one, oral rivaroxaban was prophylactically administered to prevent venous thrombus embolism (VTE). All patients were encouraged to start early limb exercises such as ankle pumps, quadriceps and gluteus isometric contractions, and flexion of knee and hip joint on the bed immediately after surgery, which was aimed to improve the range of motion and prevent VTE. Patients were recommended to undergo examinations with an X-ray for postoperative six weeks, three months, six 
months, one year. Then they underwent clinical and imaging examinations once yearly until the end of the follow-up.

\section{Statistical Methods}

The data are presented as the mean and standard deviation. The unpaired t-test and Fisher's exact test was used to analyze continuous variables between groups, and chi-square test was used for count data comparisons. Kaplan-Meier survivorship analyses were conducted with revision for any reason and revision for aseptic loosening as the endpoints. Statistical analyses were performed with the use of SPSS Statistics software version 26.0 .

\section{Results}

106 patients were alive with an average of $62.1 \pm 8.3$ months follow-up, 29 patients died during a 0.5-39 months follow-up period. The one-year mortality of cemented and uncemented group was $12.9 \%$ and $6.1 \%$, respectively $(p=0.048)$. Demographic and clinical characters of patients including gender, age, BMl, ASA grade, Evans-Jensen classification, the Singh index and the preoperative hemoglobin between cemented and uncemented groups were compared with no significant differences observed (Table 1). Detail data on intraoperative and postoperative outcomes was listed (Table 2). Significant differences regarding the estimated blood loss, the amount of transfusion and the duration of the operation were observed ( $p<0.05$, Table 2). Finally, 36 patients in group $A$ and 35 patients in group B can walk independently without any aid. Three patients in group A and four patients in group $B$ lied in bed due to cerebral infarction or hip fracture. No patients died due to the operation, and no intraoperative femur fracture occurred. 
Table 1

Demographic and clinical characters of patients

\begin{tabular}{|llll|}
\hline & $\begin{array}{l}\text { Cemented group } \\
(\mathbf{n}=\mathbf{7 0})\end{array}$ & $\begin{array}{l}\text { Cementless group } \\
(\mathbf{n}=\mathbf{6 5})\end{array}$ & P value \\
\hline Gender (Male/Female) & $28 / 42$ & $19 / 46$ & 0.19 \\
\hline Age at the surgery ${ }^{1}$ (year) & $84.9 \pm 4.0$ & $85.0 \pm 4.1$ & 0.80 \\
\hline BMI ${ }^{1}$ & $23.3 \pm 3.4$ & $22.3 \pm 2.9$ & 0.34 \\
\hline ASA grade & & & 0.05 \\
\hline II & 38 & 29 & \\
\hline III & 26 & 35 & 0.49 \\
\hline IV & 6 & 1 & \\
\hline Evans-Jensen classification & & 15 & 0.19 \\
\hline III & 13 & 25 & 0.31 \\
\hline IV & 23 & 25 & \\
\hline V & 34 & $2.7 \pm 0.5$ & \\
\hline Singh index & & \\
\hline Preoperative hemoglobin (g/L) & $102.6 \pm 19.3$ & $101.9 \pm 16.6$ & \\
\hline 1The values were given as the mean and standard deviation & \\
\hline
\end{tabular}


Table 2

Intraoperative and postoperative outcomes of patients

\begin{tabular}{|c|c|c|c|}
\hline & $\begin{array}{l}\text { Cemented group } \\
(n=70)\end{array}$ & $\begin{array}{l}\text { Cementless group } \\
(n=65)\end{array}$ & $P$ value \\
\hline Operative time ${ }^{1}(\mathrm{~min})$ & $52.1 \pm 6.4$ & $37.9 \pm 4.5$ & 0.03 \\
\hline Blood loss $^{1}(\mathrm{ml})$ & $431.4 \pm 40.1$ & $379.2 \pm 52.2$ & 0.04 \\
\hline Transfusion & & & 0.01 \\
\hline No. of patients & 42 & 33 & \\
\hline No. of units ${ }^{1}$ & $3.8 \pm 2.4$ & $3.0 \pm 1.2$ & \\
\hline Weight training time ${ }^{1}(\mathrm{~d})$ & $2.9 \pm 0.7$ & $3.0 \pm 0.7$ & 0.34 \\
\hline Hospital stay length after surgery ${ }^{1}(d)$ & $6.4 \pm 8.8$ & $4.6 \pm 1.4$ & 0.06 \\
\hline Postoperative ambulatory status & & & 0.78 \\
\hline Walk without any aid & 36 & 36 & \\
\hline Walk independently with any aid & 13 & 16 & \\
\hline Lie in bed & 3 & 5 & \\
\hline Postoperative mortality & & & 0.19 \\
\hline Within 30 days postoperatively & 3 & 0 & \\
\hline Within one year postoperatively & 9 & 4 & \\
\hline At $1-4$ year postoperatively & 6 & 7 & \\
\hline Overall mortality & 18 & 11 & 0.21 \\
\hline Harris hip score ${ }^{1}$ at the last follow-up & $77.6 \pm 11.6$ & $77.3 \pm 10.2$ & 0.14 \\
\hline
\end{tabular}

In group A, one patient had a superficial infection at the postoperative two weeks, underwent a debridement surgery and was cured. One patient had a dislocation at two months after surgery and was cured with closed reduction and abduction bracing for one month without a recurrence. One patient had the contralateral hip fracture due to slip down, and was treated with conservative treatment because of poor general condition and senile dementia. In group $B$, one patient had a dislocation at four months after surgery and was cured with closed reduction and abduction bracing for one month without a recurrence. In group B periprosthetic fractures occurred in two patients within one year after surgery due to slip down, 
and they were treated with conservative treatment due to poor general condition. No symptomatic deep vein thrombosis or pulmonary embolism occurred in all patients.

Radiological signs of bone ingrowth were observed in all cementless femoral stems at the final follow-up. Radiological evidence of loosening, acetabular erosion and acetabular migration were not detected in all patients (Fig. 1 and Fig. 2). Trochanteric nonunion was observed in five patients. 12 hips were observed with grade I heterotrophic ossification. Around the stem tip mild cortical porosis was detected. Although two patients had periprosthetic fractures, they refused revision due to poor general condition. At the last follow-up, no patients underwent femoral revision due to any reason.

\section{Discussion}

Our study revealed that in comparison with cemented hemiarthroplasty, cementless hemiarthroplasty using a long modular stem without reconstruction of femoral calcar can provide satisfactory clinical outcomes and function scores. Besides, the duration of surgery and estimated blood loss were less in cementless group.

For unstable intertrochanteric fractures, the representative treatment is internal fixation including intramedullary fixation (Gamma nail, proximal femoral nail anti-rotation) and plate fixation (dynamic hip screw, dynamic condylar screw). Intertrochanteric fractures mainly occur in the elderly, who often have osteoporosis, comorbidities, poor bone healing and postoperative complications, and these patients have high mortality[23, 24]. Internal fixation is difficult to maintain stable reduction for unstable intertrochanteric fractures in the elderly due to osteoporosis, varus deformity, cut-out, malunion, and metal failure, and patients are recommended to restrict early weight wearing and prolong bed rest, thus resulting in related complications[18]. So bipolar hemiarthroplasty is preferred in elderly intertrochanteric fractures and has advantages of early ambulation due to the immediate stability[8], however, cementation of acrylic cement can cause cardiopulmonary complications[11,25]. Cementless bipolar hemiarthroplasties can avoid the side effect caused by cement. Several studies have reported the use of cementless stems in elderly intertrochanteric fractures; to gain the initial stability and early ambulation, the long stems have been applicated[9, 10,13,17]. To reconstruct the femoral calcar, it will need a surgeon with a wealth of surgical skills, extra expose and cerclage wires for reduction and fixation of the fragment, which will cause more time cost and blood loss, thus increasing the risk for octogenarians. Abdelkhalek et al. reported that the mean operative time was $140 \mathrm{~min}$ (range 110-170 min) for unstable intertrochanteric fractures with the femoral calcar reconstruction[26]. Park et al. reported that the mean operative time was $75.3 \mathrm{~min}$ (range 50-185 $\mathrm{min}$ ) for unstable intertrochanteric fractures treated with a rectangular cross-section stem[27]. Kim et al. reported that the mean operative time of cementless calcarreplacement hemiarthroplasty for unstable intertrochanteric fractures is $96 \pm 26 \mathrm{~min}$ [13]. In our study, the mean operative time without femoral calcar reconstruction was $37.9 \pm 4.5 \mathrm{~min}$, which is more less than previous reports. And compared with the cemented group, the mean operative time was less $(p<0.01)$. 
Due to the distal rigid fixation through MPIl, patients with cementless stems can achieve initial stability and walk with a crutch at a mean $3.0 \pm 0.7$ days. Porous-coated stem can induce bone ingrowth and reduce the risk of loosening. Besides, the femur was hand-reamed so no intraoperative femur fractures occurred in our patients. With the proximal components and spacers, the stem can fit the leg length and femoral anteversion better. Given all this, even the femoral calcar was not reestablished, patients with MPII prostheses still achieved enough initial stabilities and satisfactory clinical functions as well as patients with cemented prostheses.

Studies on the bone cement implantation syndrome have been reported. Olsen et al. conducted a retrospective cohort study on 1095 patients with femoral neck fractures treated with cemented hemiarthroplasty $(n=986)$ and uncemented hemiarthroplasty $(n=109)$, the incidence of hypoxia and/or hypotension was higher in the cemented group $(p=0.003)$, the incidence of severe hypotension/hypoxia was $6.9 \%$ in the cemented group while no severe hypotension/hypoxia was observed in the uncemented group[28]. Tan et al. retrospectively investigated the on-table, day zero, day one, day seven, and day 30 mortality of 751 femoral neck fracture patients with cemented stems, 7-day and 30-day mortality rates were $2.7 \%$ and $6.8 \%$, respectively. Compared with total hip arthroplasty, patients with hemiarthroplasty have a higher 30-day mortality associated with increasing ASA grade $(p<0.01)$ when adjusted for age, gender, and type of surgery[29]. Duijnisveld et al. reported that 9-year mortality rates did not differ between patients with femoral neck fractures undergoing cemented or uncemented hemiarthroplasties, 9year revision rates were $3.1 \%$ (cemented group) and $5.1 \%$ (uncemented group), respectively $(p<0.05)[30]$. Fenelon et al. conducted a meta-analysis on the perioperative mortality after cemented or uncemented hemiarthroplasties for displaced femoral neck fractures, 22 studies with a total of 183167 hemiarthroplasties were reviewed, the cemented hemiarthroplasty is associated with an increased mortality rate for the first 48 hours $(p<0.01)$, and no difference was observed for day seven, day 30 and one-year mortality[31]. To avoid the bone cement implantation syndrome for very elderly patients with intertrochanteric fractures, a long cementless stem is a good choice. Kim et al. have proved the satisfactory mid-term outcomes with $97.3 \%$ survival rate for any reason and $99.1 \%$ survival rate for femoral stem revision[32]. In our study, compared with cemented group, patients in cementless group showed no significant differences in postoperative ambulatory status, overall mortality and HHS. However, within postoperative 30 days, three patients in cemented group died while no patient died in cementless group. Periprosthetic fractures occurred in two patients within one year after surgery in cementless group, in consistent with the study by Yoon, B. et al., we should pay attention to prevent periprosthetic fractures especially during one-year postoperatively for cementless stems[33]. In our study, the one-year mortality of cemented and uncemented group was $12.9 \%$ and $6.1 \%$, respectively $(p=0.048)$. Our one-year mortality in uncemented group was less than previously reported one-year mortality[13, 18].

This study has several limitations. First, it did not include a large number of patients. However, in this study we only focused on patients aged 80 years or more treated with cemented or uncemented hemiarthroplasty without calcar reconstruction for unstable intertrochanteric fractures, which makes eligible patients less. Second, the follow-up period was relatively short. In fact, a long-term follow-up is 
barely possible in octogenarians with the mean age of 84.9 years and the overall mortality of $21.5 \%$ in our study. And it has limited clinical relevance considering the remaining life expectancy.

\section{Conclusions}

In conclusion, compared with cemented hemiarthroplasty, cementless hemiarthroplasty using a long modular stem without reconstruction of femoral calcar was a good option for very elderly patients with unstable intertrochanteric fractures, which is proved safety, early stability and less cost of operative time.

\section{Abbreviations}

ASA: American Society of Anesthesiologist; BMI: body mass index;

HHS: Harris hip score; VTE: venous thrombus embolism

\section{Declarations}

Ethics approval This retrospective study does not contain any experimental intervention on human participants or animals and thus meets all ethical standards described in the Declaration of Helsinki.

Consent to participate In this retrospective study, informed consents were obtained from all patients.

Consent for publication Though all patient information is blinded, in our study, all parents gave their written informed consent to publish the obtained data of this study.

Availability of data and materials The datasets generated or analysed during the current study are included in the published article as well as they are available from the corresponding author on reasonable request.

Competing interests All authors declare that they have no conflict of interest.

Funding This work was supported by National Natural Science Foundation of China [grant number 81972044].

Authors' contributions Chang Sun, Jianning Zhao and Ting Guo conceived and designed the study. Ting Guo and Jianning Zhao performed the surgery. Chang Sun, Meng Cai, Jia Meng, and Hui Jiang collected the data, analyzed the data, carried out the statistical analysis and wrote the manuscript. Jianning Zhao and Ting Guo assisted in discussion of the results and writing up.

All authors read and approved the final manuscript.

Acknowledgements The authors would like to thank all participating patients and study nurses who made this retrospective study possible. 


\section{References}

1. Alexiou KI, Roushias A, Varitimidis SE. et al(2018)Quality of life and psychological consequences in elderly patients after a hip fracture: a review. CLIN INTERV AGING. 13: 143-150. doi: 10.2147/CIA.S150067.

2. Zhou S, Liu J, Zhen P. et al(2019)Proximal femoral nail anti-rotation versus cementless bipolar hemiarthroplasty for unstable femoral intertrochanteric fracture in the elderly: a retrospective study. BMC Musculoskelet Disord. 20(1): 500. doi: 10.1186/s12891-019-2793-8.

3. Haidukewych GJ, Israel TA, Berry D. J(2001)Reverse obliquity fractures of the intertrochanteric region of the femur. J BONE JOINT SURG AM. 83(5): 643-650. doi: 10.2106/00004623-200105000-00001.

4. Kashigar A, Vincent A, Gunton MJ. et al(2014)Predictors of failure for cephalomedullary nailing of proximal femoral fractures. BONE JOINT J. 96-B(8): 1029-1034. doi: 10.1302/0301620X.96B8.33644.

5. Yoo JH, Kim TY, Chang JD. et al(2014)Factors influencing functional outcomes in united intertrochanteric hip fractures: a negative effect of lag screw sliding. ORTHOPEDICS. 37(12): e1101e1107. doi: 10.3928/01477447-20141124-58.

6. Socci AR, Casemyr NE, Leslie MP, et al(2017)Implant options for the treatment of intertrochanteric fractures of the hip: rationale, evidence, and recommendations. BONE JOINT J. 99-B(1): 128-133. doi: 10.1302/0301-620X.99B1.BJJ-2016-0134.R1.

7. Mäkinen TJ, Gunton M, Fichman SG, et al(2015)Arthroplasty for Pertrochanteric Hip Fractures. ORTHOP CLIN N AM. 46(4): 433-444. doi: 10.1016/j.ocl.2015.06.001.

8. Chan KC, Gill G. S(2000)Cemented hemiarthroplasties for elderly patients with intertrochanteric fractures. Clin Orthop Relat Res. (371): 206-215. doi: 10.1097/00003086-200002000-00025.

9. Choy WS, Ahn JH, Ko JH, et al(2010)Cementless bipolar hemiarthroplasty for unstable intertrochanteric fractures in elderly patients. Clin Orthop Surg. 2(4): 221-226. doi: 10.4055/cios.2010.2.4.221.

10. Lee YK, Ha YC, Chang BK. et al(2011)Cementless bipolar hemiarthroplasty using a hydroxyapatitecoated long stem for osteoporotic unstable intertrochanteric fractures. J ARTHROPLASTY. 26(4): 626-632. doi: 10.1016/j.arth.2010.05.010.

11. Parvizi J, Ereth MH, Lewallen D G(2004)Thirty-day mortality following hip arthroplasty for acute fracture. J BONE JOINT SURG AM. 86(9): 1983-1988. doi: 10.2106/00004623-200409000-00017.

12. Hossain M, Andrew J. G(2012)Is there a difference in perioperative mortality between cemented and uncemented implants in hip fracture surgery? INJURY. 43(12): 2161-2164. doi: 10.1016/j.injury.2012.08.043.

13. Kim SY, Kim YG, Hwang JK(2005)Cementless calcar-replacement hemiarthroplasty compared with intramedullary fixation of unstable intertrochanteric fractures. A prospective, randomized study. $\mathrm{J}$ BONE JOINT SURG AM. 87(10): 2186-2192. doi: 10.2106/JBJS.D.02768. 
14. Cankaya D, Ozkurt B, Tabak A. Y(2013)Cemented calcar replacement versus cementless hemiarthroplasty for unstable intertrochanteric femur fractures in the elderly. Ulus Travma Acil Cerrahi Derg. 19(6): 548-553. doi: 10.5505/tjtes.2013.57615.

15. Kim Y, Moon JK, Hwang KT, et al(2014)Cementless bipolar hemiarthroplasty for unstable intertrochanteric fractures in octogenarians. Acta Orthop Traumatol Turc. 48(4): 424-430. doi: 10.3944/AOTT.2014.13.0119.

16. Gocer $\mathrm{H}$, Coskun $\mathrm{S}$, Karaismailoglu $\mathrm{N}$. Comparison of treatment of unstable intertrochanteric fracture with different arthroplasty methods. Niger Med J. 2016;57(2):81-5. doi:10.4103/0300-1652.182081.

17. Wada K, Mikami H, Oba K, et al(2017)Cementless calcar-replacement stem with integrated greater trochanter plate for unstable intertrochanteric fracture in very elderly patients. J Orthop Surg (Hong Kong). 25(1): 612345037. doi: 10.1177/2309499016684749.

18. Zha GC, Liu J, Wang Y. et al(2019)Cementless distal fixation modular stem without reconstruction of femoral calcar for unstable intertrochanteric fracture in patients aged 75 years or more. Orthop Traumatol Surg Res. 105(1): 35-39. doi: 10.1016/j.otsr.2018.11.015.

19. Singh M, Nagrath AR, Maini PS(1970)Changes in trabecular pattern of the upper end of the femur as an index of osteoporosis. J BONE JOINT SURG AM. 52(3): 457-467. doi.

20. Engh CA, Massin P, Suthers KE1990)Roentgenographic assessment of the biologic fixation of porous-surfaced femoral components. Clin Orthop Relat Res. (257): 107-128. doi.

21. Capello WN, D'Antonio JA, Geesink RG, et al(2009)Late remodeling around a proximally HA-coated tapered titanium femoral component. Clin Orthop Relat Res. 467(1): 155-165. doi: 10.1007/s11999008-0550-7.

22. Meijerink HJ, Gardeniers JW, Buma P, et al(2004)Hydroxyapatite does not improve the outcome of a bipolar hemiarthroplasty. Clin Orthop Relat Res. (421): 143-150. doi: 10.1097/01.blo.0000126307.64581.c6.

23. Gaumetou E, Zilber S, Hernigou. P(2011)Non-simultaneous bilateral hip fracture: epidemiologic study of 241 hip fractures. Orthop Traumatol Surg Res. 97(1): 22-27. doi: 10.1016/j.otsr.2010.07.011.

24. LeBlanc ES, Hillier TA, Pedula KL, et al(2011)Hip fracture and increased short-term but not long-term mortality in healthy older women. Arch Intern Med. 171(20): 1831-1837. doi: 10.1001/archinternmed.2011.447.

25. Grimsrud C, Monzon RJ, Richman J. et al(2005)Cemented hip arthroplasty with a novel cerclage cable technique for unstable intertrochanteric hip fractures. J ARTHROPLASTY. 20(3): 337-343. doi: 10.1016/j.arth.2004.04.017.

26. Abdelkhalek M, Ali AM, Abdelwahab. M(2013)Cemented bipolar hemiarthroplasty with a cerclage cable technique for unstable intertrochanteric hip fractures in elderly patients. Eur J Orthop Surg Traumatol. 23(4): 443-448. doi: 10.1007/s00590-012-1006-z.

27. Park MS, Cho HM, Kim JH. et al(2013)Cementless bipolar hemiarthroplasty using a rectangular cross-section stem for unstable intertrochanteric fractures. HIP INT. 23(3): 316-322. doi: 10.5301/hipint.5000024. 
28. Olsen F, Hard ASM, Nellgard B. et al(2020)The role of bone cement for the development of intraoperative hypotension and hypoxia and its impact on mortality in hemiarthroplasty for femoral neck fractures. ACTA ORTHOP. 91(3): 293-298. doi: 10.1080/17453674.2020.1745510.

29. Tan KG, Whitehouse SL, Crawford RW(2020)On-Table and Short-Term Mortality: A Single-Institution Experience With Cementing All Hip Arthroplasties for Neck of Femur Fractures. J ARTHROPLASTY. 35(4): 1095-1100. doi: 10.1016/j.arth.2019.11.027.

30. Duijnisveld BJ, Koenraadt K, van Steenbergen LN. et al(2020)Mortality and revision rate of cemented and uncemented hemiarthroplasty after hip fracture: an analysis of the Dutch Arthroplasty Register (LROI). ACTA ORTHOP. 91(4): 408-413. doi: 10.1080/17453674.2020.1752522.

31. Fenelon C, Murphy EP, Pomeroy E. et al(2020)Perioperative Mortality After Cemented or Uncemented Hemiarthroplasty for Displaced Femoral Neck Fractures-A Systematic Review and Meta-analysis. The Journal of Arthroplasty. doi: 10.1016/j.arth.2020.08.042.

32. Kim J, Kim H, Kim J, et al(2018)Mid-Term Survivals After Cementless Bipolar Hemiarthroplasty for Unstable Intertrochanteric Fractures in Elderly Patients. The Journal of Arthroplasty. 33(3): 777-782. doi: 10.1016/j.arth.2017.10.027.

33. Yoon B, Lee Y, Jo W, et al(2016)Incidence and Risk Period of Periprosthetic Femoral Fracture After Cementless Bipolar Hemiarthroplasty in Elderly Patients. The Journal of Arthroplasty. 31(6): 13261330. doi: 10.1016/j.arth.2015.12.030.

\section{Figures}
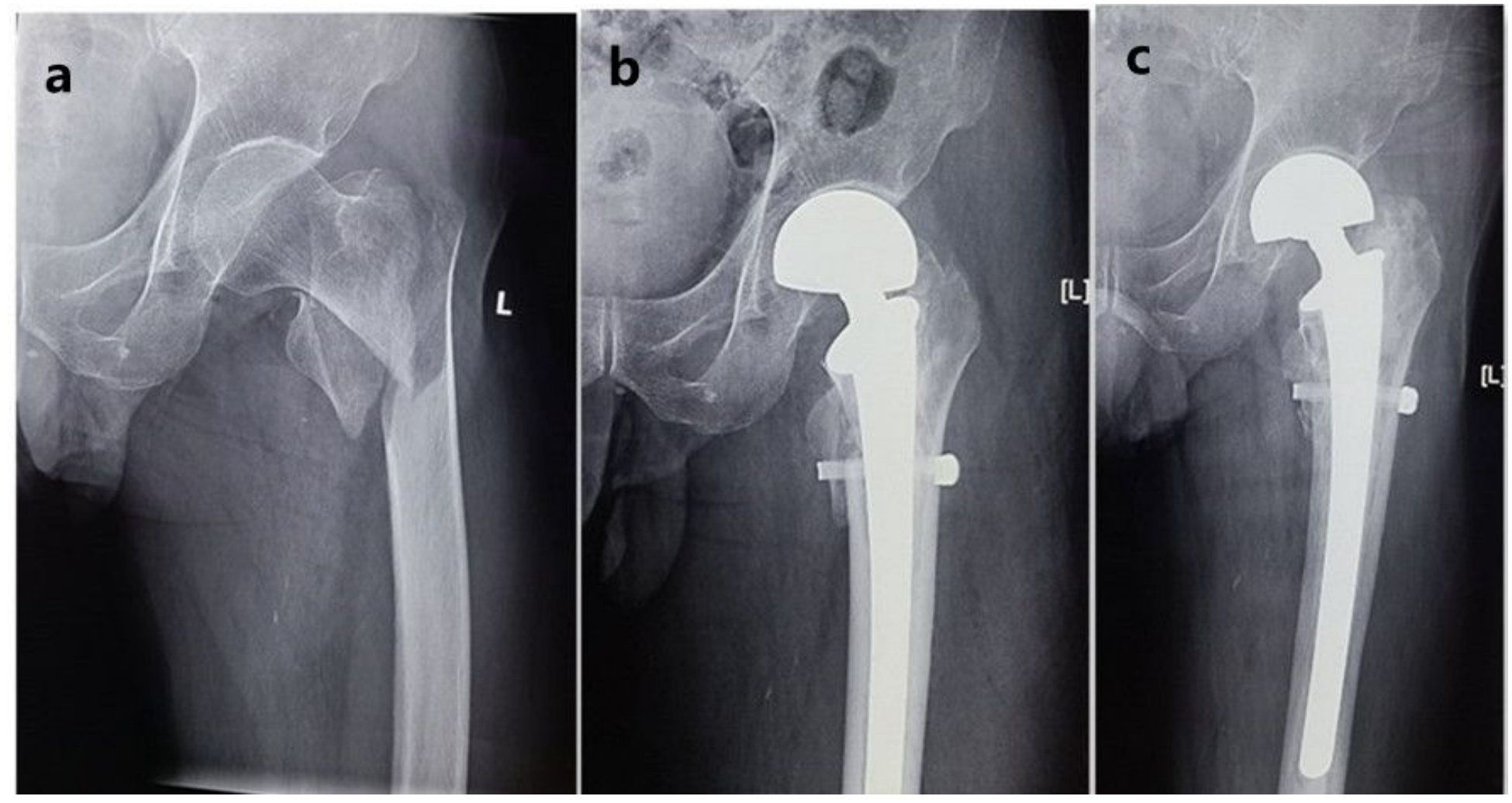

Figure 1 
80-year-old man with an unstable intertrochanteric fracture of the left hip. (a) Preoperative anteroposterior view (b) Postoperative radiographic image. He underwent cemented hemiarthroplasty with the lesser trochanter reduced and fixed. (c) The postoperative 6-year follow-up view showed well-fixed stem and bony union of the lesser trochanter.

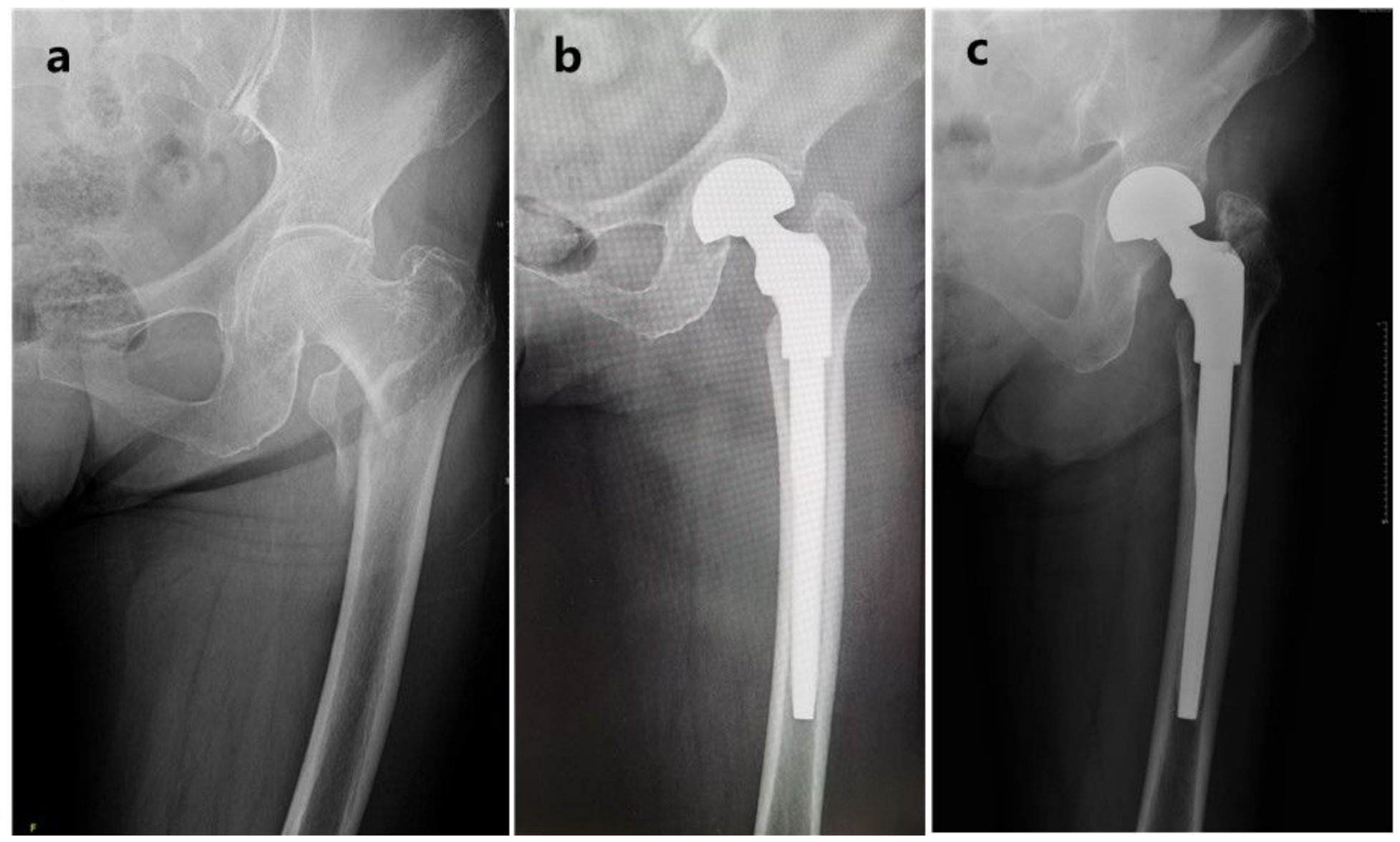

Figure 2

90-year-old woman with an unstable intertrochanteric fracture of the left hip. (a) Preoperative anteroposterior view (b) Postoperative radiographic image. She underwent cementless hemiarthroplasty with the lesser trochanter removed, and the greater trochanter with no obvious displacement was not fixed with cerclage wires. (c) The postoperative 4-year follow-up radiograph showed no osteolysis and subsidence of femoral stem except nonunion of the greater trochanter. 